\title{
Dissociative identity disorder and prepulse inhibition of the acoustic startle reflex
}

\author{
Karl Yngvar Dale' \\ Magne Arve Flaten' \\ Åke Elden' \\ Arne Holte ${ }^{2}$ \\ 'Department of Psychology, University \\ of Troms $\varnothing$, Norway; ${ }^{2}$ The Norwegian \\ Institute of Public Health, Department \\ of Mental Health, Oslo, Norway and \\ University of Oslo, Norway
}

\begin{abstract}
A group of persons with dissociative identity disorder (DID) was compared with a group of persons with other dissociative disorders, and a group of nondiagnosed controls with regard to prepulse inhibition (PPI) of the acoustic startle reflex. The findings suggest maladaptive attentional processes at a controlled level, but not at a preattentive automatic level, in persons with DID. The prepulse occupied more controlled attentional resources in the DID group compared with the other two groups. Preattentive automatic processing, on the other hand, was normal in the DID group. Moreover, startle reflexes did not habituate in the DID group. In conclusion, increased PPI and delayed habituation is consistent with increased vigilance in individuals with DID. The present findings of reduced habituation of startle reflexes and increased PPI in persons with DID suggest the operation of a voluntary process that directs attention away from unpleasant or threatening stimuli. Aberrant voluntary attentional processes may thus be a defining characteristic in DID.
\end{abstract}

Keywords: dissociation, DID, PPI, startle, habituation

\section{Introduction}

Dissociation is regarded to reflect an ability to protect oneself from intrusive stressors by perceptual detachment (van der Kolk et al 1996; Nijenhuis et al 1998; Perry and Pollard 1998). In this study, prepulse inhibition (PPI) of the startle reflex, which may index both automatic and controlled processing, was investigated in individuals with dissociative disorders and dissociative identity disorder (DID). The ability to protect oneself from stressors, hypothesized to be accentuated in high dissociators, should be related to prepulse inhibition. The present study investigated whether this protection occurred at a preattentive automatic level, or whether it could best be understood as a controlled voluntary process.

Dissociation involves alterations in consciousness, depersonalization and fragmentation of memory and sense of self. DID is characterized by the presence of two or more distinct identities or personality states, each with its own relatively enduring pattern of perceiving, relating to, and thinking about the environment and self. The disturbance is not due to the direct physiological effects of a substance (APA 1994).

Waller and colleagues (1996) identified two types of dissociation, pathological and nonpathological, by using taxometric analyses. These two types of dissociation do not exist along a continuum; rather they constitute two distinguishable latent classes (Meehl and Golden 1982). The taxometric studies seem to strongly support the validity of a pathological dissociative disorder whose features are consistent with that of DID (Gleaves et al 2001).

Severe, chronic childhood trauma is considered to be the main etiological factor in the development of pathological dissociative conditions (Boon and Draijer 1993; Kirby et al 1993; Nijenhuis et al 1998; Gleaves et al 2001). Patients with dissociative problems usually suffer the full spectrum of symptoms related to the diagnosis 
of post-traumatic stress disorder (PTSD; Kirby et al 1993; APA 1994; Nijenhuis et al 1998).

Only a few psychophysiological studies have been conducted on dissociative phenomena. In an experiment involving a series of repetitions of a startle eliciting stimulus, Ladwig and colleagues (2002) found that high-level dissociative patients with PTSD, compared with high-level dissociative patients without PTSD, showed increased startle reflexes and delayed habituation, indicative of increased arousal in these patients. These data suggest that dissociative disorders are not associated with increased physiological reactivity. In fact, dissociative disorders seem to be associated with reduced physiological reactivity. Griffin and colleagues (1997), using heart rate and skin conductance as measures, found that there was significantly more suppression of autonomic physiological responses among high dissociators compared with low dissociators when interviewed about previous rape episodes. Ebner-Premier and colleagues (2005) found smaller startle reflexes in borderline disorder patients with high dissociation compared to borderline patients with low dissociation. One possible mechanism that could explain reduced physiological reactivity in dissociative disorders is reduced attention to external stimuli. Kirino (2006) examined the pathophysiology of dissociative phenomena using the P300 component of event-related potentials, an index of controlled attentional processing, and found that patients with dissociative diagnoses exhibited attenuation of P300 amplitudes during dissociative episodes when compared with controls, indicative of reduced attention to external stimuli, but exhibited recovery to control levels in remission. The data from Kirino (2006) suggest impaired attention in DID, but only during dissociative episodes. However, that study did not use stimuli that could be classified as threatening. Taken together, these studies are consistent with the hypothesis that dissociative disorders are characterized by defensive processes in the form of reduced sensory intake or impaired attention to external stimuli.

Attentional processing of a stimulus first engages preattentive mechanisms, ie, automatic, reflex-like orienting, detection, and analysis, that allows the assessment of whether the stimulus is important and in need of further processing. If the stimulus is considered important or relevant, attention is directed to the stimulus by controlled or voluntary processes. Nijenhuis and colleagues (1998) claimed that dissociative defensive reactions are elicited almost instantaneously, implying that preattentive processing is sufficient for the expression of the defensive reactions and that the reactions occur without conscious control. In the present study, this hypothesis was investigated by the method of prepulse inhibition (PPI) of the acoustic startle reflex.

The startle response is a reflex that occurs among most humans and animals in reaction to an abrupt, strong sensory stimulus, for instance a loud noise. The magnitude of this response exhibits several forms of plasticity, eg, PPI. PPI refers to attenuation in response to a strong stimulus (pulse) if this is preceded shortly by a weak nonstartling stimulus (prepulse). It provides an operational measure of sensorimotor gating that serves to prevent the interruption of ongoing perceptual and early sensory analysis. Prepulse inhibition is hypothesized to reflect an automatic preattentive inhibitory process that functions to protect the initial processing of the prepulse by dampening the effects of other concurrent or immediately following events such as a startle stimulus (Graham 1975). Prepulse inhibition deficits have mainly been studied by presenting weak acoustic stimuli prior to intense startle-eliciting noise. As the participant orients to the prepulse to process it at a preattentive level, processing of other competing input is inhibited and the amplitude of the startle reflex is consequently reduced (Graham 1975). When the interval between the weak stimulus (the prepulse) and the reflex-eliciting stimulus is approximately 30 to about $300 \mathrm{~ms}$ there is reliable reduction of the startle reflex amplitude compared to when the reflex is elicited in the absence of the prepulse (Elden and Flaten 2002, 2003).

PPI has been shown to be amplified by controlled attentional processes, since directing attention towards the prepulse inhibits startle even further (Blumenthal and Flaten 1994). When participants are instructed to monitor the prepulse for an extended time period, increased PPI is often seen at approximately $400 \mathrm{~ms}$ compared to a control condition where participants are not monitoring the prepulse (Elden and Flaten 2002, 2003). Thus, voluntarily directing attention to the prepulse inhibits startle reflexes for an extended period of time after prepulse onset.

In the present study PPI was tested at stimulus onset asynchronies (SOAs) between the prepulse and the startleeliciting stimulus of 30 to 420 milliseconds. Three groups were employed; a group diagnosed with DID, a group with other dissociative disorders, and a group of normal controls. Preattentive processes were tested in a condition where PPI was tested without any requirements (no-task), whereas controlled processes were introduced in a separate condition by instructing the participants to judge the duration of the stimuli presented (task). If patients with dissociative disorder have reduced sensory intake or impaired attention to external stimuli (Kirino 2006), they should show impaired PPI. If the deficits in 
attentional processing occurred at a preattentive level, reduced PPI should be seen in both the task and no-task conditions, at the 30 to $120 \mathrm{~ms}$ stimulus onset asynchronies (SOAs). At the 150 and $420 \mathrm{~ms}$ SOAs, reduced PPI would be indicative of impaired controlled attention directed to the prepulse.

Based on findings by Ebner-Priemer and colleagues (2006), it was also predicted that high dissociators should show smaller startle reflexes to the startle-eliciting stimulus presented alone. This would be consistent with the defensive reaction of reduced attention to external stimuli.

\section{Method}

\section{Participants}

Three groups of participants were recruited in order to represent three populations including persons with DID, persons with other dissociative disorders, and nondiagnosed persons. A total of 29 persons participated in the study. Participants in the DID sample (the DID group) consisted of 8 women, with a mean age of 34.1 and an age range from 21 to 46 . All of these participants had been hospitalized at least once due to their mental disorder. Only two were currently in treatment at a psychiatric ward and six attended outpatient clinics. Two participants were chronically disabled and received welfare, three participants were in a rehabilitation program including work training, two were on medical leave of absence from their daytime jobs, and one participant was working $80 \%$ of full-time. Participants in the DID group came from all over Norway and were recruited via a nationwide search including 46 psychiatric institutions, both outpatient clinics and wards. The institutions were contacted directly by telephone and letter stating the intentions of the study. The institutions were encouraged to pass on our quest to any patient they might be in contact with who had been diagnosed with DID. If any of these persons were interested to participate in the study, they were encouraged by their therapists to verify this to the first author (KYD) by telephone, email, or a written letter.

Participants in the dissociative disorders sample (the DD group) consisted of 7 women and one man with a mean age of 33.7 and an age range from 19 to 45 . Two participants in this sample were diagnosed with depersonalization disorder only and the remaining six participants were diagnosed with both depersonalization disorder and dissociative amnesia. Three participants in this group were chronically disabled, two were in a rehabilitation program, one was a college student, and two participants worked full-time. All participants in this group had been hospitalized due to their mental disorder. One was currently receiving treatment at a ward and seven received treatment at outpatient facilities. Most of the participants in the DD group were recruited by contacting nearby mental care institutions using the same procedure as that was used to recruit persons with DID. Some participants were also recruited via a newspaper advertisement. In the advertisement, which was run in the local newspapers of Tromsø and Stavanger, it was stated that we were interested in recruiting persons who either had a diagnosis of DID or who had some of the following experiences: 1) Finding themselves in a place and having no idea how they got there; 2) Not being able to remember important life events; 3) Acting so differently in different situations that they feel as if they were different persons; and 4) Hearing voices inside their head that tell them what to do.

The nondiagnosed sample (the CONTROL group) consisted of 7 women and 6 men with a mean age of 29.1 and an age range from 20 to 41 . None of these had dissociative disorders and none were currently in treatment for mental health problems or substance abuse or had histories of such treatment. Eleven persons were university students and two worked part-time within the framework of a work related rehabilitation program. Participants in the CONTROL group were mostly recruited through an e-mail recruitment campaign at the University of Tromsø. Some of the participants were also recruited via a newspaper advertisement in the local newspaper in Tromsø in which it was stated that people were needed to participate in clinical and psychophysiological tests.

All participants had auditory thresholds of $20 \mathrm{~dB}$ or less in both ears at $1000 \mathrm{~Hz}$. Seven of the participants in the DID group, three of the participants in the DD group and one participant in the CONTROL group used a prescription drug on a regular basis. The medication in the DID group consisted of centralstimulants (two participants), antidepressants (two participants), anxiolytics (two participants), a thyreoid hormone drug (one participant), a hypnotic agent (one participant), and an antipsychotic drug (one participant). In the DD group anxiolytics were used by three participants and one participant used an antidepressant. None of the participants in the CONTROL group used any drug.

The research was approved by the Regional Committee for Medical Research Ethics in Health Region V in Norway, and was conducted according to the Helsinki declaration. Written informed consent was obtained from all participants. No monetary reward was given to the participants.

\section{Clinical measures}

\section{Assessment of dissociative diagnosis}

Clinical assessment with regards to dissociative diagnosis was obtained through the administration of the SCID-D 
(Steinberg 1995). The SCID-D is a 276 item structured clinical interview used to diagnose DSM -IV dissociative disorder. The SCID-D also includes registration of demographic data, work history, treatment history, somatic disease, substance abuse and family history. The schedule has an overall interrater reliability of 0.68 (Kappa), a sensitivity of $90 \%$, and a specificity of $100 \%$ for the diagnosis of DID (Steinberg 1995). A SCID-D interview usually takes approximately 90 minutes.

\section{Assessment of current and lifetime traumatic stress}

Current and lifetime traumatic stress was measured with the CAPS (Clinician-Administered PTSD Scale; Blake et al 1997) that is a structured interview designed to assess 17 symptoms of PTSD outlined in the DSM-IV. Scores are only attained when subjects report having experienced one or more traumatic event (Criterion A in the DSM-IV diagnosis). The CAPS can be used to assess the severity and frequency of each symptom. It provides a comprehensive assessment of both lifetime and current PTSD. Frequency and intensity ratings are made on five-point scales. A CAPS interview usually lasts from 40 to 90 minutes depending on the extensiveness of the traumatic experiences.

\section{Assessment of dissociative level}

Dissociative level was measured with the Dissociative Experience Scale (DES; Bernstein and Putnam 1986), The DES is a 28-item self-report questionnaire that has been reported to be reliable, internally consistent, and temporally stable (Dubester and Braun 1995). It is not a diagnostic tool but serves as a screening device for dissociative disorders. Participants are required to circle the percentage of time (given in increments of $10 \%$ ranging from $0-100$ ) that they have the kind of experience described in each item. A total score is computed as the mean of the responses to the 28 items. High dissociators will usually be identified among those with mean scores of 30 or above (Bernstein and Putnam 1986).

\section{Assessment of subjective states}

The mood rating scale of Bond and Lader (1974) was used to assess alertness, calmness, and contendness.

\section{Apparatus and stimuli}

The experiment was conducted in an electrically and sound shielded chamber (Tegnér) where temperature was maintained at $20 \pm 1.5^{\circ} \mathrm{C}$. Control of the experiment and data acquisition was performed via a Keithley 575 interface. All programs for experimental control and data scoring were written in ASYST 3.1 by the second author (MAF).
The auditory equipment used for the hearing test was a Grason-Stadler, Inc. GSI 17 Audiometer (accuracy $\pm 3 \%$ ) and Telephonix TDH39 earphones with MX41AR cushions. Background noise levels were ambient at about $28 \mathrm{~dB}$ (Flaten et al 2005).

The startle-eliciting noise had an intensity of $95 \mathrm{~dB}$ SPL with instantaneous rise time and duration of $50 \mathrm{~ms}$, and was produced by a Coulbourn S81-02 noise generator. The associated comparison stimulus had an intensity of $85 \mathrm{~dB}$ SPL with instantaneous rise-time and a duration of 40 or $60 \mathrm{~ms}$. The output was passed to a Coulbourn S77-06 multiplier/divider and then to a Coulbourn S78-03 linear summing amplifier. The output was sent to a NAD Electronics 3225PE stereo amplifier and then to a pair of Telephonix TDH 39 earphones. The tone prepulse stimuli and the associated comparison stimuli had intensities of $60 \mathrm{~dB}$ SPL with rise times of $20 \mathrm{~ms}$, a frequency of $1000 \mathrm{~Hz}$, and were generated by a Coulbourn S81-06 signal generator, the output of which was sent to a Coulbourn S84-04 rise/fall gate. The signal then entered a Coulbourn linear summing amplifier, then the NAD amplifier, and finally the earphones.

Eye blink EMG reflexes were recorded from the left orbicularis oculi with $\mathrm{Ag} / \mathrm{AgCl}$ Beckman miniature electrodes filled with TECA conducting paste. The EMG signal was amplified with a factor of 60,000 and filtered (passing 90-250 Hz) by a Coulbourn S75-01 Bioamplifier. The signal was rectified and integrated by a Coulbourn S76-01 contour-following integrator with a $10 \mathrm{~ms}$ time constant, and the output was sent to the computer via the Keithley interface. Sampling on each trial began $200 \mathrm{~ms}$ prior to onset of the first stimulus and continued for $200 \mathrm{~ms}$ after onset of the startle stimulus. The sampling rate was $10 \mathrm{~Hz}$ prior to onset of the first stimulus and $1000 \mathrm{~Hz}$ after stimulus onset.

Comparison stimuli were presented $4000 \mathrm{~ms}$ prior to the prepulse and startle-eliciting stimuli. Half of the comparison stimuli were $10-20 \mathrm{~ms}$ shorter than the prepulse and startle stimulus, and the other half of the comparison stimuli were 10-20 ms longer than the prepulse and startle stimulus. The duration of the prepulse was 30,60, 90, 120,150 , or $420 \mathrm{~ms}$, while the duration of the associated comparison stimulus was 20 or 40,40 or 80,70 or 110,100 or 140,130 or 170 , and 400 or $440 \mathrm{~ms}$, respectively. The duration of the startle stimulus was $50 \mathrm{~ms}$ and the associated comparison stimulus was $\pm 10-20 \mathrm{~ms}$. Prior to electrode placement the skin was cleaned with pads containing alcohol and pumice. The EMG electrodes were attached about 10-15 $\mathrm{mm}$ below the pupil and about 15-20 mm 
below the outer canthus of the left eye. A ground electrode was placed on the forehead.

\section{Procedure}

The whole test program lasted a day including intermissions during which the participants filled in the DES form, underwent clinical assessment with the two clinical interviews, the SCID-D and CAPS (performed successively in that order), and lastly, were tested in the psychophysiological laboratory. The clinical interviews were administered by a research assistant who had been trained specifically for this occasion. The laboratory procedures were administered by the first author (KYD).

All participants were examined under identical laboratory conditions. The duration of the experiment was approximately $66 \mathrm{~min}$. Participants were tested under two experimental conditions involving both a passive (No-task) and an active (Task) attention protocol. Subjective arousal and mood was measured before, between and after the protocols.

At the beginning of each experimental session the participants were placed in an armchair and told that the purpose of the experiment was to investigate effects of attention on physiological and psychological reflexes. Electrodes for measurement of EMG and earphones were then placed on the participants. Lastly, participants were instructed to stay awake, sit with their eyes open, and move as little as possible during the experiment.

Each experiment involved presentations, in a pair-wise fashion, of continuous pure-tone prepulses followed by a white noise startle-eliciting stimulus. The stimulus onset asynchronies (SOAs) between the prepulse and the startle stimulus were 30,60, 90, 120, 150, and $420 \mathrm{~ms}$. Each of the six SOAs was presented 12 times in semi-random order. The startle stimulus was also presented alone 12 times. Thus 84 trials were presented in each of the Task and No-task conditions. The intertrial interval varied between 13-24 s with a mean of $18 \mathrm{~s}$. The seven conditions were presented in nine blocks of eight trials, one trial for each condition. This ensured random presentation of the SOAs and the control condition. In the Task condition, participants were asked to compare the duration of the tone and noise in each stimulus pair. The following information was provided to the participants before the test: "You will hear some tones and some scratch noises. First a tone is presented and shortly afterwards a scratch is presented. Four seconds later, the same pair of stimuli will be presented a second time. Your task is to judge whether the duration of the second tone is shorter or longer than the duration of the first tone, and if the second scratch is longer or shorter than the first scratch." A similar procedure was used by Heekeren and colleagues (2004) where participants were instructed to direct their attention both to the prepulse and the pulse. In the No-Task condition the participants did not receive any instructions besides to sit down, relax, and keep their eyes open; the condition was otherwise identical with the Task condition. The order of presentation of the Task and No-task conditions were counterbalanced across subjects. After completion of the experimental procedure the electrodes were removed.

\section{Reflex scoring and data treatment}

The reflex was scored 20-120 ms after onset of the startle stimulus. To count as a startle reflex, the integrated EMG voltage had to increase at least $30 \mathrm{~A} / \mathrm{D}$ units relative to baseline, which was computed as the mean EMG in the $200 \mathrm{~ms}$ prior to stimulus onset. Reflex amplitude was the maximum difference between baseline EMG level and peak, in arbitrary analog-to-digital units. Prepulse inhibition was calculated as the ratio of reflex amplitudes on prepulse trials to startle stimulus alone trials. The proportion measure recommended by Blumenthal and colleagues (2004) is less dependent on control startle amplitude.

The subjective indexes of arousal and mood were expressed as posttest minus pretest scores.

\section{Design and analysis}

The design was a 3 -Group (DID, DD, and CONTROL $) \times 2$ Task (Task, No-task) $\times 6$ SOA $(30,60,90,120,150,420 \mathrm{~ms})$ mixed design with the first factor treated as a between-participants factor and the two last factors treated as within-participants factors. Subjective arousal and mood were analyzed as a 3 Group by 3 Test (before, between the conditions, after) mixed design. The data were analyzed by analysis of variance and significant effects were followed-up with contrast analyses. An alpha level of 0.01 was used when the presence of PPI was tested and multiple contrasts were computed. Effect sizes were computed as $\eta^{2}$. To ascertain that PPI was observed, t-tests of differences from 0 (no PPI) were performed for each Group at each SOA.

\section{Results \\ Subjective arousal and mood}

There was a main effect of Test for alertness $(\mathrm{F}(2,90)=31.08$, $\left.\mathrm{p}<0.0001, \eta^{2}=0.40\right)$ due to decreased alertness across Tests for all Groups. No main effects or interactions were significant for the contentedness and calmness dimensions of the Bond and Lader (1974) scale. 


\section{Current and life time PTSD}

There were several reports among the participants in the DID and DD groups of both sexual and physical abuse during adulthood and childhood and sexual and physical assault by a close relative during childhood. Participants in these groups had also experienced a wide range of other traumatic incidents, such as serious illness, natural disaster and dramatic accidents. There were no reports in the CONTROL group of experiences related to nonintrusive, nonabusive incidents, or to experiences related to sexual abuse or physical assault. All participants in the DID group met the criteria for PTSD diagnosis, both current and life time. In the DD group, six met the criteria for current PTSD and seven met the criteria for life time PTSD. None of the participants in the CONTROL group met either of these criteria. As shown in Table 1, only the two clinical groups attained PTSD scores. The scores are a summation of trauma-related symptoms and the intensity level for each symptom. The DID group scored higher than the DD group, both with regards to current and life time PTSD, but these differences were not significant.

\section{Dissociative level}

Dissociative levels (Table 1) as measured by the DES varied slightly but not significantly across the clinical groups with mean scores of 43.00 in the DID group and 40.50 in the DD group. The CONTROL group had a mean score of 10.42 and scored significantly lower than both the DID group $(\mathrm{F}(1,26)=14.77, \mathrm{p}=0.001)$ and the DD group $(\mathrm{F}(1,26)=$ $12.59, \mathrm{p}=0.002)$.

\section{Startle alone}

Reflexes to the noise alone habituated across Trials in the CONTROL and DD groups but not in the DID group (Group by Trials interaction $\left(\mathrm{F}(22,286)=1.82, \mathrm{p}<0.005, \eta^{2}=0.10\right)$. This was confirmed by trend analyses that showed linear trends in the CONTROL and DD groups $(\mathrm{F}(1,26)=7.25$ and 4.62, respectively, ps $<0.05$ ), but not in the DID group

Table I Mean scores and standard deviations for the DID, DD, and CONTROL groups on the DES and the CAPS reflecting dissociative level and current/life time PTSD, respectively.

\begin{tabular}{llll}
\hline Measures & $\begin{array}{l}\text { DID } \\
\text { Mean (N) SD }\end{array}$ & $\begin{array}{l}\text { DD } \\
\text { Mean (N) SD }\end{array}$ & $\begin{array}{l}\text { CONTROL } \\
\text { Mean (N) SD }\end{array}$ \\
\hline DES & $43.00(8) 25.70$ & $40.50(8) 20.00$ & $10.42(13) 8.31$ \\
Current PTSD & $76.75(8) 10.11$ & $44.87(8) 38.52$ & $0.00($ (13) 0.00 \\
Life time PTSD & $97.50(8) 8.14$ & $82.00(8) 33.70$ & $0.00($ I3) 0.00 \\
\hline
\end{tabular}

$(\mathrm{F}<2, \mathrm{p}=0.18)$ (Figure 1). There was also a significant interaction of Task by Trials $(\mathrm{F}(11,286)=2.88, \mathrm{p}<0.002$, $\left.\eta^{2}=0.09\right)$ due to more pronounced habituation in the Task condition.

\section{PPI}

To ascertain that PPI was in fact observed, t-tests from 0 were performed. For the CONTROL group, significant inhibition of startle was seen at the $30,60,90,120$ and $150 \mathrm{~ms}$ SOAs (ts $(12)<-4.70$, ps $<0.001$ ) and not at the $420 \mathrm{~ms} \mathrm{SOA}$ $(\mathrm{t}(12)=-1.80, \mathrm{p}=0.11)$. The same pattern was seen in the DD group, where significant inhibition was seen at the SOAs from 30 to $150 \mathrm{~ms}$ (ts $(7)<-4.25$, ps $<0.004)$, whereas there was no inhibition at the $420 \mathrm{~ms} \mathrm{SOA}(\mathrm{t}(7)=-2.06$, $\mathrm{p}=0.08)$. In the DID group, however, significant PPI was seen at all SOAs (ts $(7)<-4.20$, ps $<0.004)$.

There were significant main effects of Task $(\mathrm{F}(1$, 26) $\left.=20.10, \mathrm{p}<0.01, \eta^{2}=0.42\right)$ due to increased PPI during the Task condition compared to the No-task condition (means of -0.62 and -0.35 , respectively). There was also a main effect of $\operatorname{SOA}\left(F(5,130)=15.71, p<0.01, \eta^{2}=0.34\right)$. This was due to increased PPI at the $90 \mathrm{~ms}$ SOA compared to the $30 \mathrm{~ms} \mathrm{SOA}$ (ps $<0.05$ ). There was also less PPI at the $420 \mathrm{~ms}$ SOA compared to the other five SOAs (ps $<0.0001$ ).

There was an interaction of Group $x \operatorname{SOA}(\mathrm{F}(10,130)=$ $\left.2.00, \mathrm{p}<0.04, \eta^{2}=0.08\right)$. Figure 2 shows that the CONTROL group displayed strong and reliable PPI. The DID group displayed somewhat weaker PPI compared to the CONTROL group at the shorter SOAs, but there was a tendency to more inhibition in the DID group compared to the

\section{Startle alone: Group by Trials}

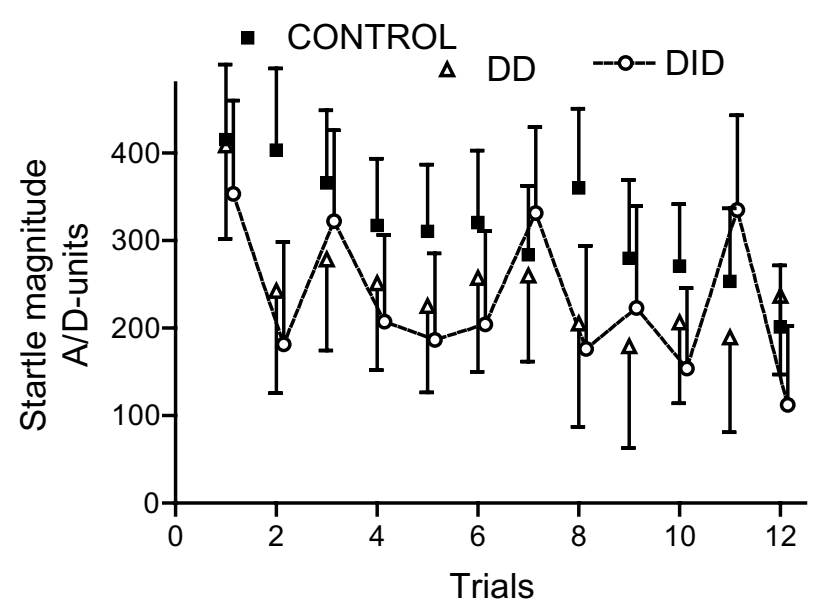

Figure I Group by Trials interaction: Mean startle reflex magnitudes across the 12 startle-alone trials for each group. Error bars indicate I standard error of the mean. 

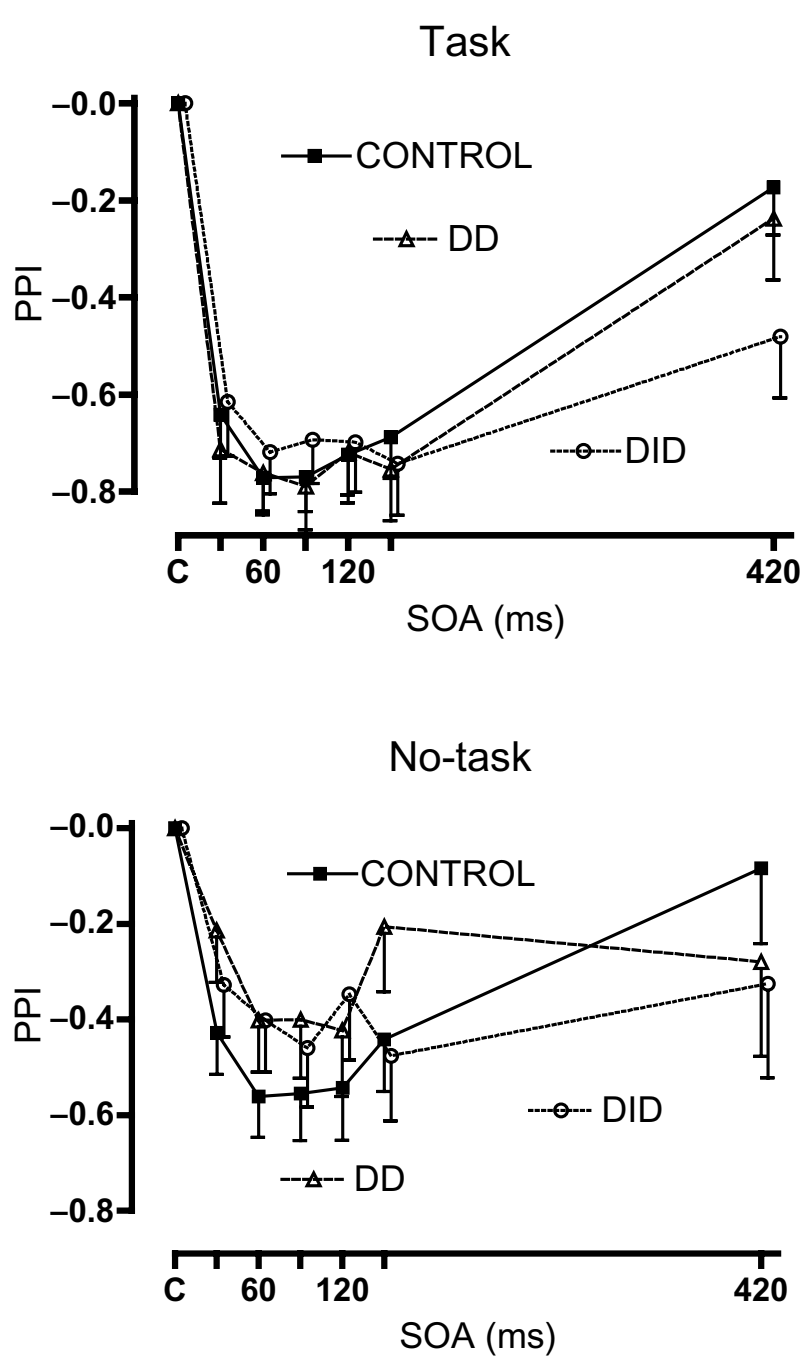

Figure 2 Group by Task by SOA interaction: Mean PPI across stimulus onset asynchronies (SOAs) in milliseconds for each group in the Task and No-task conditions. $C$ refers to startle alone control levels. Error bars indicate I standard error of the mean.

CONTROL group at the $420 \mathrm{~ms} \mathrm{SOA}$ in the Task condition $(\mathrm{p}=0.064)$. The DID group did not display the hyperbolic PPI function usually observed at the present SOAs. This was confirmed in a trend analysis where significant quadratic trends were seen in the CONTROL and DD groups ( $\mathrm{Fs}(1$, $26)=36.53$ and 10.66 , respectively, ps $<0.01)$, but not in the DID group $(\mathrm{F}=3.38, \mathrm{p}=0.077)$.

Finally, the interaction of Task by $\operatorname{SOA}(\mathrm{F}(5,130)=2.97$, $\mathrm{p}<0.02, \eta^{2}=0.09$ ) was due to increased PPI in the Task compared with the No-task condition at the $30,60,90,120$, and $150 \mathrm{~ms}$ SOAs (ps $<0.0001)$. No other main effects or interactions were significant $(\mathrm{F}<1)$.

\section{Discussion}

Startle reflexes to the startle-eliciting stimulus alone were of the same magnitude in all three groups. Thus, any dif- ferences in PPI could not be attributed to differences in startle magnitudes. Moreover, by presenting the PPI data as proportion of difference from control, the effect of between-group differences in startle magnitudes on PPI were reduced or eliminated (Blumenthal et al 2004).

The interaction of Group by Trials in the startle alone data was due to a lack of habituation in the DID group. Figure 1 shows that responding in the DID group was variable across Trials compared to the DD and CONTROL groups where habituation was orderly across Trials. This was supported by significant linear trends in the CONTROL and DD groups, but not in the DID group. A lack of habituation indicates heightened vigilance in the DID group as these participants did not inhibit the response to the intense noise bursts as was observed in the other two groups. Reduced habituation of the startle reflex has been related to hypervigilance (Orr et al 2002). It might also be explained in accordance with Davidson and colleagues (2004) as a disability to classify loud noises as redundant information and hence stop reacting to them. The present data suggests that such an underlying pathology is present in DID, but not necessarily in other dissociative disorders.

The variability in startle reflexes across trials in the DID group could be related to the order of presentation of stimuli. Startle-eliciting stimuli presented alone were interleaved with prepulse trials in the present experiment. Thus, dishabituation, ie, the recovery of a habituated reflex due to presentation of a novel stimulus, probably played a role in the results and mostly so in the DID group. According to the classic habituation theory of Groves and Thompson (1970), dishabituation reflects the general process of sensitization or arousal, and this seems to have been increased in the DID group.

There was significant PPI in all three groups. The interaction of Group by SOA, however, indicated aberrant PPI among participants diagnosed with DID compared to nondiagnosed controls. At the $420 \mathrm{~ms}$ SOA, PPI in the DID group did not return to baseline levels as it did in the group of healthy volunteers and the DD group. This was supported by significant quadratic trends in the DD and CONTROL groups, but not in the DID group. Thus, the prepulse seems to have occupied controlled attentional resources for a longer time period in the DID group compared to the other two groups. The data from the CONTROL and DD groups indicate that these groups analyzed the prepulse, as evidenced by significant PPI, and then shifted their attention away from the prepulse, as evidenced by a return of startle reflex magnitudes, to baseline levels. Inhibition of startle reflexes at $420 \mathrm{~ms}$ indicates that attention was not shifted away from the 
prepulse in the DID group, and that the prepulse still occupied resources in this group. This finding is highly unusual and indicates extended processing of prepulses in the DID group. One possible explanation is that significant PPI at the $420 \mathrm{~ms}$ SOA reflects an inhibitory process that protects the individual from an intrusive stressor, ie, the startle-eliciting stimulus. This has been described as a defining characteristic of dissociation (van der Kolk et al 1996; Nijenhuis et al 1998; Perry and Pollard 1998).

Closer scrutiny of the PPI data from the DID group reveals somewhat reduced but still normal PPI in this group for the first $120 \mathrm{~ms}$ after prepulse onset. Maximum PPI was seen at $90 \mathrm{~ms}$, which is normal with acoustic stimuli at the stimulus intensities used in the present study, and slightly decreased PPI was seen at 120 compared to $90 \mathrm{~ms}$. However, at $150 \mathrm{~ms}$, PPI again increased in the DID group. It is interesting that the "break" in the normal PPI function occurs between 120 and $150 \mathrm{~ms}$, when stimulus processing is assumed to shift from automatic to controlled processes (Dawson et al 1997). Thus, it seems that preattentive automatic processing was normal in the DID group, but that more controlled attentional processing was directed to the prepulse compared with the two other groups. Thus, the PPI data support the conclusion that the protective process hypothesized to inhibit the effect of intrusive stressors in high dissociators is a controlled voluntary process, and does not occur at an automatic preattentive level as hypothesized by Nijenhuis and colleagues (1998).

How reduced habituation of startle in the DID group relates to increased voluntary attention to the prepulse remains to be answered. Reduced habituation may indicate that the DID group found the startle stimulus more unpleasant. Since the prepulse signaled the occurrence of the startleeliciting noise, the prepulse could have allowed individuals in the DID group to direct their attention elsewhere, ie, to the prepulse with a consequent inhibition of startle reflexes. It is therefore suggested that this voluntary direction of attention away from the intrusive stressor reflects a defining characteristic in DID.

Differences in startle or PPI could not be attributed to arousal, dissociative level or gender. General arousal, as assessed by the Bond and Lader scale (1974), did not vary across Groups and regarding dissociative level, there was only a minor difference between the two clinical groups. There were relatively more males in the Control group compared with the other two groups, and prepulse inhibition has been shown to be sensitive to menstrual cycle at SOAs of $120 \mathrm{~ms}$ and shorter (Jovanovic et al 2004). In the present study a between-group difference was seen at $420 \mathrm{~ms}$, whereas there were no differences between the groups at the shorter SOAs. Thus, the gender of the participants was most likely not the reason for the accentuated PPI in the DID group at $420 \mathrm{~ms}$. A separate ANOVA that only included women confirmed this.

The DID group was more distressed than the DD group in terms of PTSD symptoms but the differences between these groups on measures of current and life time PTSD were not statistically significant. This may rule out the possibility that the differences in startle and PPI between the DID and the DD groups are attributable to degrees of PTSD-severity. Rather, we may infer that these differences are related to the division between pathological and nonpathological dissociation (Waller et al 1996), with the DD group reflecting the nonpathological category of dissociation and the DID group reflecting the pathological.

We must be cautious in our generalizations primarily due to the small samples in the two clinical groups and secondarily, because the nondissociative comparison group was not optimally matched to the clinical groups in terms of medication. Furthermore, participants were screened for psychiatric co-morbidity and substance abuse only within the framework of the SCID-D and not by the use of a more extensive assessment tool for co-morbidity. In addition, all participants joined the study through self-selection. Also, the different samples were recruited by different means. For example, the DID group was mainly recruited via direct contact with mental care institutions and the CONTROL group was recruited via a newspaper advertisement and email. Even though there are no obvious reasons why this should make a difference for the results, these issues should be considered in future studies. Lastly, there may have been underreported levels of anxiety and distress in the CONTROL group. The CAPS, basically mirroring the structure of the PTSD diagnosis as defined in the DSM-IV, is not a very "finemasked" instrument for measuring traumatic exposure. Scores are only attained when subjects report having experienced one or more traumatic event (reflecting Criterion A in the DSM-IV diagnosis), which none of the participants in the CONTROL group did. Optimally, we could have used an assessment tool that captured stress-related symptoms irrespective of traumatic incidents.

In summary, the present findings of reduced habituation of startle reflexes and increased PPI in persons with DID suggest the operation of a voluntary process that directs attention away from unpleasant or threatening stimuli. Aberrant voluntary attentional processes may thus be a defining 
characteristic in DID. In future studies it might be advisable to use longer SOAs than the $420 \mathrm{~ms}$ used in this study to investigate the time-course of PPI in persons with DID. Any tendencies detected in the present experiment might be more pronounced in response to threatening stimuli. It may be considered to use threatening stimuli as prepulses or to use fear conditioning procedures as in Davis and colleagues (1989) or Sasaki and Hanamoto (2007). After fear conditioning, individuals with DID should attend to the conditioned stimulus, and this could increase its impact on the processing of the startle stimulus.

In clinical terms, our findings support the notion that persons with DID consciously redefine their perceptions of the environment when this environment starts to become unpleasant and intrusive. In this process the individual seems to change point of view on demand and is able to alter his or her experience in the situation by rerouting the perception of the stimuli observed.

A growing body of research is emerging that deals directly with decomposing the psychophysiological mechanisms of DID (Reinders et al 2003; Williams et al 2003). Undoubtedly, persons with this condition have a unique sense of the world and unique cognitive capabilities. In order to understand these capabilities, we will need to combine a wide variety of experimental and clinical research. Ultimately, this will have important clinical implications, given that more precise knowledge about pathological dissociation and DID with regards to clinical, cognitive and psychophysiological features can ensure that more precise therapeutic judgments are made.

\section{Acknowledgment}

The present study was funded by grants from the North Norwegian Centre of Psychiatric Research. The study was performed while the first and third authors were $\mathrm{PhD}$ students at the University of Tromsø.

\section{References}

Allen JJ, Movius HL. 2000. The objective assessment of amnesia in dissociative identity disorder using event-related potentials. Int $J$ Psychophysiol, 38:21-41.

[APA] American Psychiatric Association. 1994. Diagnostic and statistical manual of mental disorders 4th ed. Washington DC: American Psychiatric Press.

Bernstein EM, Putnam FW. 1986. Development reliability and validity of a dissociation scale. J Nerv Ment Dis, 174:727-35.

Blake DD, Weathers FE, Nagy LM, et al. 1997. Clinician-administered PTSD scale for DSM-IV Current and lifetime diagnostic version. National Center for Post Traumatic Stress Disorder.

Blumenthal TD, Elden Å, Flaten MA. 2004. A comparison of several methods used to quantify prepulse inhibition of eyeblink responding. Psychophysiol, 41:326-32.
Blumenthal TD, Flaten MA. 1994. Selective effects of attentional direction on the startle reflex at different stages of processing. Psychobiol, 22:338-46.

Bond A, Lader M. 1974. The use of analogue scales in rating subjective feelings Br J Med Psychol, 47:211-18.

Boon S, Drajer N. 1993. Multiple personality disorder in the Netherlands: a clinical investigation of 71 patients. Am J Psychiatry, 150:489-94.

Braff DL, Geyer MA, Swerdlow NR. 2001. Human studies of prepulse inhibition of startle: Normal subjects patient groups and pharmacological studies. Psychopharmacology, 156:234-58.

Butler RW, Braff DL, Rausch JL, et al. 1990. Physiological evidence of exaggerated startle response in a subgroup of Vietnam veterans with combat-related PTSD. Am J Psychiatry, 147:1308-12.

Davidson JRT, Stein DJ, Shalev AY, et al. 2004. Posttraumatic stress disorder: Acquisition recognition course and treatment. J Neuropsychiatry Clin Neurosci, 16:135-47.

Davis M, Schlesinger LS, Sorenson CA. 1989. Temporal specificity of fear conditioning: Effects of different conditioned stimulus-unconditioned stimulus intervals on the fear-potentiated startle effect. J Exp Psychol Anim Behav Process, 15:295-310.

Dawson ME, Schell AM, Swerdlow NR, et al. 1997. Cognitive clinical and neurophysiological implications of startle modification. In: Lang PJ, Simons RF, Balaban M (eds). Attention and orienting. London: Lawrence Erlbaum, pp. 257-79.

Dubester KA, Braun BG. 1995. Psychometric properties of the Dissociative Experience Scale. J Nerv Ment Dis, 183:231-5.

Ebner-Priemer UW, Badeck S, Beckmann C, et al. 2005. Affective dysregulation and dissociative experience in female patients with borderline personality disorder: a startle response study. $J$ Psychiatr Res, 39:85-92.

Elden Å, Flaten MA. 2002. The relationship of automatic and controlled processing to prepulse inhibition. $J$ Psychophysiol, 16:46-55.

Elden Å, Flaten MA. 2003. Similar effects of attention directed to acoustic and tactile stimuli on prepulse inhibition of acoustic startle. Scand $J$ Psychol, 44:363-72.

Filion DL, Dawson ME, Schell AM. 1998. The psychological significance of human startle eyeblink modification: a review. Biol Psychol, 47:1-43.

Flaten MA, Nordmark E, Elden Å. 2005. Effects of background noise on the human startle reflex and prepulse inhibition. Psychophysiol, 42:298-305.

Gleaves DH, May MC, Cardena E. 2001. An examination of the diagnostic validity of dissociative identity disorder. Clin Psychol Rev, 21:577-608.

Graham FK. 1975. The more or less startling effects of weak prestimulation Psychophysiol, 12:238-48.

Greaves GB. 1980. Multiple personality 165 years after Mary Reynolds. J Nerv Ment Dis, 168:577-96.

Griffin MG, Resick PA, Mechanic MB. 1997. Objective assessment of peritraumatic dissociation: Psychophysiological indicators. Am J Psychiatry, 154:1081-8.

Grillon C, Morgan CA, Southwick SM, et al. 1996. Baseline startle amplitude and prepulse inhibition in Vietnam veterans with posttraumatic stress disorder. Psychiatry Res, 64:169-78.

Groves PM, Thompson RF. 1970. Habituation: A dual process theory. Psychol Rev, 77:419-50.

Hamm AO, Cuthbert BN, Globisch J, et al. 1997. Fear and the startle reflex: Blink modulation and autonomic response patterns in animal and mutilation fearful subjects. Psychophysiol, 34:97-107.

Heekeren K, Meincke U, Geyer MA, et al. 2004. Attentional modulation of prepulse inhibition: a new startle paradigm. Neuropsychobiology, 49:88-93.

Jovanovic T, Szilagyi S, Chakravorty S, et al. 2004. Menstrual cycle effects on prepulse inhibition of acoustic startle. Psychophysiol, 41:401-6.

Kirby JS, Chu JA, Dill DL. 1993. Correlates of dissociative symptomatology in patients with physical and sexual abuse histories. Compr Psychiatry, 34:258-63. 
Kirino E. 2006. P300 is attenuated during dissociative episodes. $J$ Nerv Ment Dis, 194:83-90.

Ladwig KH, Marten-Mittag B, Deisenhofer I, et al. 2002. Psychophysiological correlates of peritraumatic dissociative responses in survivors of life-threatening cardiac events. Psychopathology, 35:241-8.

Meehl PE, Golden R. 1982. Taxometric methods. In: Kendall P, Butcher $\mathrm{J}$ (eds). Handbook of research methods in clinical psychology. New York: Wiley, pp. 127-81.

Nijenhuis ERS, Vanderlinden J, Spinhoven P. 1998. Animal defensive reactions as a model for trauma-induced dissociative reactions. J Trauma Stress, 11:243-60.

Orr SP, Metzger LJ, Pitman RK. 2002. Psychophysiology of post-traumatic stress Disorder. Psychiatr Clin North Am, 25:271-93.

Perry BD, Pollard R. 1990. Homeostasis stress trauma and adaptation: A neurodevelopmental view of childhood trauma. Child Adolesc Psychiatr Clin N Am, 7:33-52.

Reinders AATS, Nijenhuis ERS, Paans AMJ, et al. 2003. One brain two selves Neuroimage, 20:2119-25.
Sasaki H, Hanamoto A. 2007. Shock sensitization and fear potentiation of auditory startle response in hamsters. Percept Mot Skills, 105:862-71.

Steinberg M. 1995. Structured clinical interview for DSM-IV dissociative disorders SCID-D. Oslo: Norsk Psykologforening.

van der Kolk BA, van der Hart O, Marmar CR. 1996. Dissociation and information processing in postraumatic stress disorder. In: van der Kolk BA, McFarlane AC, Weisaeth L (eds). Traumatic stress: The effects of overwhelming experience on mind body and society. New York: Guilford Press.

Waller NG, Putnam FW, Carlson EB. 1996. Types of dissociation and dissociative types: A taxometric analysis of dissociative experiences. Psychol Methods, 1:300-21.

Williams CL, Haines J, Sale IM. 2003. Psychophysiological correlates of dissociation in a case of dissociative identity disorder. J Trauma Dissociation, 4:101-17. 